\title{
Letters
}

DOI:10.2214/AJR.07.2318

WEB - This is a Web exclusive article.

\section{Real-Time Elastography in the Assessment of Liver Fibrosis}

We read with great interest the article by Friedrich-Rust et al. [1] in the March 2007 issue of $A J R$ and would like to raise a number of important issues in relation to their work.

First, in this study [1] the aspartate transaminase-to-platelet ratio index (APRI) test was highly sensitive and more accurate than the elasticity score in evaluating liver fibrosis. Moreover, combining the APRI test with realtime elastography did not seem to significantly increase the diagnostic accuracy. The results of this study show that, taking into account the cost-effectiveness issue, the APRI test is preferable to real-time elastography because it is less expensive and more accurate.

Second, to add information from blood markers the authors [1] combined the elasticity score with the platelet count and gammaglutamyl transpeptidase (GGT) without considering age and cholesterol, even though all four variables are analyzed in the Forns index used to evaluate liver fibrosis [2].
Third, the authors [1] performed real-time elastography twice in 16 different patients with liver disease to assess the reproducibility of different variables characterizing elasticity. It would be interesting to know the results. Moreover, how was the intra- and interobserver variability of this new technique assessed? Specifically, this study did not measure within- and between-session intraobserver reliability and within-session interobserver reliability. Sonography is an operator-dependent technique. We are currently working on a new real-time elastography machine. In our experience, different levels of training and experience could also affect the results of real-time elastography. It would be interesting to know how the authors dealt with this issue. Were all the examinations performed by the same operator?

Fourth, in the evaluation of real-time elastography, four patients without clinical or biochemical evidence of cirrhosis, staged with METAVIR fibrosis score F4 by liver histology, were combined with 20 patients in whom biopsy was not performed because they had obvious clinical signs of liver cirrhosis [1]. This group of patients is not homogeneous because the early stage of liver cirrhosis is dif- ferent than the advanced stage, in which there is progress in the fibrotic process [3].

And last, to determine the accuracy of elasticity scores for correct prediction of liver fibrosis stage, patients staged with METAVIR fibrosis score F2 should be analyzed separately from patients staged with METAVIR fibrosis score F3.

Giovanna Ferraioli

Rosario Gulizia

Carlo Filice

IRCC S. Matteo-University of Pavia

Pavia, Italy

\section{References}

1. Friedrich-Rust M, Ong MF, Herrmann E, et al. Real-time elastography for noninvasive assessment of liver fibrosis in chronic viral hepatitis. AJR 2007; 188:758-764

2. Forns X, Ampurdanès S, Llovet JM, et al. Identification of chronic hepatitis $\mathrm{C}$ patients without hepatic fibrosis by a simple predictive model. Hepatology 2002; 36[4 Pt 1]:986-992

3. Murata K, Akashio K, Ochiai Y. Changes in acidic glycosaminoglycan components at different stages of human liver cirrhosis. Hepatogastroenterology 1984; 31:261-265 